Please do not remove this page

RMIT

UNIVERSITY

\title{
How specific is domain specificity: Does it extend across playing position?
}

Bruce, Lyndell; Farrow, Damian; Raynor, Annette

https://researchrepository.rmit.edu.au/esploro/outputs/9921858574501341/filesAndLinks?institution=61RMIT_INST\&index=null

Bruce, L., Farrow, D., \& Raynor, A. (2012). How specific is domain specificity: Does it extend across playing position? Journal of Science and Medicine in Sport, 15(4), 361-367.

https://doi.org/10.1016/j.jsams.2011.11.255

Document Version: Accepted Manuscript

Published Version: https://doi.org/10.1016/j.jsams.2011.11.255

Repository homepage: https://researchrepository.rmit.edu.au

(C) 2011 Published by Elsevier Ltd on behalf of Sports Medicine Australia

Downloaded On 2023/04/26 20:34:44 +1000 
Thank you for downloading this document from the RMIT Research Repository.

The RMIT Research Repository is an open access database showcasing the research outputs of RMIT University researchers.

RMIT Research Repository: http://researchbank.rmit.edu.au/

\section{Citation:}

Bruce, L, Farrow, D and Raynor, A 2012, 'How specific is domain specificity: Does it extend across playing position?', Journal of Science and Medicine in Sport, vol. 15, no. 4, pp. 361-367.

See this record in the RMIT Research Repository at:

http://researchbank.rmit.edu.au/view/rmit:18563

Version: Accepted Manuscript

Copyright Statement: ㄷ 2011 Published by Elsevier Ltd on behalf of Sports Medicine Australia

Link to Published Version:

http://dx.doi.org/10.1016/j.jsams.2011.11.255

\section{PLEASE DO NOT REMOVE THIS PAGE}


${ }^{a}$ Australian Institute of Sport, Leverrier Crescent, Bruce, ACT, Australia - Skill Acquisition

${ }^{\mathrm{b}}$ University of South Australia, North Terrace, Adelaide, SA, Australia - School of Health Sciences

${ }^{\mathrm{e}}$ Edith Cowan University, Joondalup Dve, Joondalup, WA, Australia - School of Exercise and Health

Corresponding author:

Lyndell Bruce, Lecturer Motor Learning \& Control, RMIT University, Plenty Road, Bundoora, VIC, Australia.

25 Abstract word count: 213

26 Number of Tables: 0

27 Number of Figures: 3 
3 Objectives: The current study sought to examine the scope of domain specificity within a sport

4 expertise context through the examination of positional specificity effects in the sport of netball.

5 Design: Skilled goalers, centre court and defenders along with less skilled (novice) participants were

6 tested on a video-based decision making task.

7 Method: Skilled and less skilled netballers completed a video-based decision making task, comprising

8 scenarios from the three different positional areas in netball (goalers, centre court and defence).

9 Participants completed the scenarios from the playing position they were most familiar with, followed 10 by the remaining two positions in a counterbalanced order.

11 Results: Analysis of the goaler and defence scenarios revealed that the skilled goalers and centre court 12 players were significantly more accurate than the novice players, whilst the skilled defenders did not 13 differ to the other three groups (skilled goalers, skilled centre court and novice). For the centre court 14 scenarios the skilled centre court players and defenders were significantly more accurate than the 15 novice players, whilst the skilled goalers did not differ to any of the groups.

16 Conclusions: Limited evidence was shown to support the theory that decision making is specific to the 17 position being played with the goalers and the centre court players most accurate on the attack and 18 centre court scenarios respectively.

19

20 Keywords: Decision making, expertise, transfer, 


\section{Introduction}

Understanding expert performance in sport has typically focused on the domain (sport)

5 specific skills possessed by experts relative to novices ${ }^{1}$. While the qualities that separate performers

6 of different skill levels are well established, less is known about the positional specificity of such

7 attributes. Pyne, Gardner, Sheehan et al. ${ }^{2}$ demonstrated physical performance differences across

8 playing positions in Australian Rules Football. Ruckmen and key position players were heavier and

9 taller but had slower sprint times compared to midfield players. When decision making skill is

considered, Williams, Ward, Ward et al. ${ }^{3}$ found that while expert defensive soccer players were more accurate than their offensive counterparts this was independent of whether the scenario was of attack or defence, suggesting an absence of positional specificity effects. However, Catteeuw, Helsen, Gils et al. ${ }^{4}$ investigation of experienced soccer referees and assistant referees demonstrated role specificity effects for a foul detection and an offside judgement task. Collectively the limited evidence presented, particularly in relation to perceptual-cognitive skills, highlights the need for further investigation of the scope of domain specificity when considered in relation to positional roles. The development of a perceptual-cognitive skill, such as position specific decision making, raises a number of different theoretical and coaching/training philosophies ${ }^{5}$. Experts acquire their perceptual-cognitive knowledge through exposure to training and competition predominantly within their chosen sport. This is consistent with notions such as deliberate practice ${ }^{6}$, where the greater time an athlete spends deliberately practicing and competing within their sport, the greater their chance of improving performance over someone who spends less time engaging in the sport. A key outcome predicted of engagement in such practice is that the underlying memory representation is specific and consequently not easily transferred from one domain to another ${ }^{7}$. Such a position is also consistent with Raab's ${ }^{5}$ Situation Model of Anticipated Response consequences of Tactical training (SMART) 
that predicts that decision making skill is based on recognition of specific situations where responses are mapped to a given game situation (e.g., specific attack-defence structure in basketball ${ }^{8}$ ).

An alternative perspective emerges from the Teaching Games for Understanding (TGFU) coaching approach that suggests perceptual-cognitive expertise is not domain specific but more transferable ${ }^{9}$. This commonly prescribed coaching model suggests tactical decision making skills can be developed such that they transfer between conceptually similar sports suggesting that such skills are domain general. The limited empirical evaluation of this prediction has demonstrated tentative support for the transfer of tactical knowledge and ensuing decision making skill for conceptually similar sports $^{10,11}$. Further, while not a direct test of the TGFU approach, Abernethy, Baker and Côté ${ }^{12}$ demonstrated that participation in other sports is not necessarily detrimental to expert performance and may in some cases result in transfer back to the athletes chosen sport. Expert netball, basketball, and field hockey players demonstrated selective transfer of pattern recall skills to a level superior to that of experienced non-experts in all three sports. In particular, expert participants were able to recall a higher percentage of defensive players which seemed to be a key contributor to this effect. Smeeton, Ward and Williams ${ }^{13}$ produced similar findings in a pattern recognition task for skilled field hockey, soccer and volleyball players although skilled volleyball players were unable to transfer their volleyball perceptual-cognitive skill to the recognition of previously viewed patterns of play from soccer and field hockey.

Consequently, examining position specific decision making skills is of interest to scientists and coaches alike and provides an ideal vehicle through which to further explore both theoretical and practical issues related to the concept of domain specificity and in turn our understanding of sport expertise. If position specificity effects are demonstrated it highlights that much of the existing expertise literature may have under-estimated the expert's advantage through presentation of stimuli that is domain general rather than position specific. While it is reasonable to presume that a player who has spent the majority of their sporting life training and playing in one positional area may not be able to transfer their skills into another position, the generalisability of this prediction is of interest to coaches looking to maximise the performance of their team through changing the positional roles of 
their team members, and equally to scientists interested in the issue of transfer. Alternatively, if position specificity effects are not found, this may provide some support for developmental approaches, such as TGFU, which emphasise transfer of decision making concepts across positions and sports ${ }^{9}$.

The current study examined positional specificity effects within the sport of netball. Netball is a relatively unique sport because players are zoned in the areas into which they can move (goaler, centre court and defence). Thus if specificity effects do occur, they would be expected in a sport such as netball as players typically only play within one region of the court and do so from a relatively early stage of their career. Skilled and novice netballers completed a video-based decision making task that presented scenarios they would commonly experience in their own position as well as the other remaining playing areas (goaler, centre court and defence). It was hypothesised, in line with previous research, that the skilled players would have greater decision making accuracy than the novice participants due to their greater netball specific experiences ${ }^{14}$. Additionally, it was hypothesised that the skilled athletes would demonstrate a position specificity bias and consequently be most accurate on the scenarios from which they are most familiar with, namely their own positional area due to the development of specific memory representations for the $\operatorname{task}^{7}$, whereas the novice participants would show no positional biases due to their limited netball experience.

$$
\begin{aligned}
& \text { Methods } \\
& \text { Forty-four skilled and novice female netballers were recruited for participation in the current } \\
& \text { study with appropriate ethical approval granted from the Australian Institute of Sport and University } \\
& \text { of South Australia ethics committees. Skilled netballers were members of the Australian open-age } \\
& \text { squad and members of the Australian } 17 \text {-and-under age talent identification squad ( } \mathrm{n}=28 \text { ) (mean age } \\
& 20.5 \pm 4.4 \text { years) and with an average of } 13.8 \pm 4.3 \text { years playing experience. Six participants were } \\
& \text { classified as centre court players and had been playing in this positional area for an average of } 14.4 \pm \\
& 4.0 \text { years. Twelve participants were classified as defenders and had been playing this positional area } \\
& \text { for an average of } 13.4 \pm 3.4 \text { years. Ten participants were classified as goalers and they had been }
\end{aligned}
$$


1 playing in this positional area for an average of $13.9 \pm 5.7$ years. Prior to specialising in their current

2 positions, the skilled participants had spent the following time (years) in other positions: centre court

$3(M=6.5 \pm 1.7$ years $)$; defenders $(M=3.0 \pm 2.3$ years $)$; and goalers $(M=4.6 \pm 1.8$ years $)$. Novice

4 participants $(\mathrm{n}=16)$ were recruited from local community social sport competitions and were required

5 to have no more than five years regional netball representative playing experience, however they were

6 able to have an unlimited number of years playing in social competitions (mean age $25.3 \pm 2.6$ years;

$7 \quad 6.1 \pm 5.2$ years playing experience). The novice players were randomly allocated to one of the three

8 playing positions as none had specific experience in any one positional area.

Skilled experienced female netballers not involved in the study were filmed acting out

scenarios reflecting commonly occurring patterns of play in netball. The skilled participants may have

known some of the actors; however they were not familiar with their playing style or background.

The scenarios were filmed using a video camera (Sony Digital HDR-FX1E) placed $1.5 \mathrm{~m}$ above the ground to simulate the perspective a player would experience in a game. Each clip was edited in consultation with an expert coach (Australian national coach) so that when footage of the clip was occluded, the player in possession of the ball had two available passing options. We did not afford goalers the option of shooting as no scenarios were occluded when a goaler was in possession of the ball inside the goal circle (however, a number of the clips comprised throw-in situations taken from inside the goal circle). We did not want a player's goal shooting ability and/or confidence (or lack of) to influence the decision made. The opportunity to score (if available) may have provided a disadvantage to the centre court players and defenders who may not be able to perform a shot on goal and thus would have selected the incorrect pass option.

In netball, the rules restrict the areas into which players can move, and as a result there are three main positional groups according to their location on the court; goalers, centre court and defenders. Scenarios were designed for each positional group. In total, 32 clips were chosen for each of the positional groups. To enable a view that is most representative of the view a player would have on court when in an attacking position, scenarios for the three playing positions were filmed from

27 different positions. The defence scenarios were filmed from a position $2 \mathrm{~m}$ behind the baseline 
directly in line with the goal post. The camera position for the centre court scenarios was $5 \mathrm{~m}$ behind and directly in line with the centre circle of the netball court, and the position for the goaler's scenarios was located in the centre circle (see Figure 1). Following filming, the scenarios were edited so that the duration of each clip was between 5-10 s, and concluded at a point where one player was in possession of the ball.

Participants completed the scenarios from each of the three playing areas (goalers, centre court and defence) completing the situations from their own playing area first, and then the remaining two positional areas were presented in a counterbalanced order across participants. No presentation order effects were observed $\left(F(2,82)=0.52, p=.60, \eta p^{2}=.01\right)$. Clips were projected (InFocus LP850, OR, USA) onto a wall (2.10 m wide x $1.15 \mathrm{~m}$ high) with participants seated $3.5 \mathrm{~m}$ away. Participants were required to imagine that they were the player in possession of the ball at the conclusion of each clip, and to write down which player they would pass the ball to within three seconds of the vision occluding (netballers must pass the ball within three seconds of receiving). Four practice trials were completed prior to the beginning of each set of test trials. Thirty-two test trials were undertaken for each of the three playing areas for a total of 96 test clips. Participants were provided with a one minute break after the first sixteen trials for each set of scenarios and a two minute break between each playing area set.

The percentage of correct responses was calculated individually for each of the three positional area clip sets. Percent response accuracy was analysed using a 4 (Group; skilled goaler, skilled centre court, skilled defender, novice) x 3 (Scenario type; goaler, centre court, defence) multiple analysis of variance (MANOVA). To follow up significant MANOVA main effects, separate univariate ANOVAs were conducted as required. Pillai's Trace $(V)$ statistic was used to interpret the 
1 MANOVA with partial eta squared $\left(\eta p^{2}\right)$ values reported as a measure of effect size. Alpha was set at $2 \mathrm{p}<0.05$ for all statistical analyses unless otherwise stated.

3

\section{Results}

The overall MANOVA $\left(F(9,120)=4.04, \mathrm{p}<0.01, \eta p^{2}=.23\right)$ revealed significant differences between group and scenario type. To follow-up the between group differences, separate univariate ANOVAs revealed that the response accuracy differed across each scenario type (goaler, $F(3,40)=$ 5.28, $\mathrm{p}<0.01, \eta p^{2}=.28$; centre court, $F(3,40)=6.51, \mathrm{p}<0.01, \eta p^{2}=.32 ;$ defence, $F(3,40)=5.99, \mathrm{p}<$ $0.01, \eta p^{2}=.31$; see Figure 2). Post hoc testing revealed that for the goaler and defence scenarios, the skilled goalers and centre court players were significantly more accurate than the novice players (skilled goalers $\mathrm{p}<0.01$ in both the goaler and defence scenarios, skilled centre court $\mathrm{p}<0.05$ in both the goaler and defence scenarios), whilst the skilled defenders did not differ to the other three groups $(\mathrm{p}>0.05)$. For the centre court scenarios the skilled centre court players and defenders were significantly more accurate than the novice players (skilled centre court $\mathrm{p}<.01$, skilled defenders $\mathrm{p}<$ $0.05)$, whilst the skilled goalers did not differ to any of the groups $(\mathrm{p}>0.05)$.

$$
\text { Insert Figure } 2 \text { about here }
$$

Additional post hoc testing revealed that overall the skilled goalers and centre court players were significantly more accurate than the novice players $(F(3,43)=9.77, \mathrm{p}<0.01)$ (see Figure 3 ). Overall, irrespective of skill, the centre court scenarios $(M=70.86 \pm 9.91 \%)$ were answered more accurately than the goaler $(t(43)=-2.80, \mathrm{p}<0.01)(M=66.03 \pm 9.66 \%)$ and defence $(t(43)=2.82, \mathrm{p}<$ 0.01) $(M=66.41 \pm 8.37 \%)$ scenarios. 
2 size of 44, an alpha value of 0.05 and a partial eta squared value of .23 (equating to a Cohen's d value 3 of $\left..80^{15}\right)$.

4

5

6

7

8

9

Insert Figure 3 about here

\section{Discussion}

To assess the effect of playing position on decision making accuracy, skilled and novice netballers were tested on decision making scenarios from the three playing areas in netball (goaler, centre court and defence). We predicted that a typical perceptual-cognitive expertise effect would be apparent, whereby the skilled players would perform in a superior fashion to the novice players.

Further, we predicted that this would be accompanied by a position specific effect where the decision making performance of the skilled players would be highest for situations from their own positional area. That is, the goalers would perform best on goaler scenarios, the centre court players on centre court scenarios, and defenders on defensive scenarios due to each playing group's development of specific memory representations unique to their playing role consistent with a domain specificity view of expertise $e^{5,7}$.

While the predicted expertise effect was found, there was limited evidence to support position specific decision making effects for the skilled players. Consistent with previous expertise research, the skilled participants were, for the most part, superior to the novice group in decision making accuracy $^{14,16,17}$. This superior performance of the skilled participants may be attributed to a number of factors, including, but not limited to; developing stronger association and retrieval structures in their long term memory through prolonged domain specific practice ${ }^{5,7,8}$.

Centre court scenarios were answered most accurately irrespective of skill level indicating these may have been easier to answer correctly than the goaler and defence clips. The centre court 
scenarios included a number of centre passes, which in netball are generally well practiced and most teams possess a number of structured plays. As a result, players were able to make a more accurate judgement than when the scenarios were extracted from the general flow of play. Another reason may be that the centre court scenarios are also central to the goaler and defence clips. That is, they occur in the middle of the court where both defenders and goalers are also required to make decisions, in addition to their respective ends. These two playing groups (goalers and defenders) may therefore be able to transfer their knowledge into a neutral position where they have some experience, as opposed to the other end of the court where they have limited experience.

The absence of any position specific effects are in contrast to Williams et al. ${ }^{3}$ who found skilled defensive soccer players were more accurate at anticipating a player's action than skilled attackers and novices irrespective of the scenario type. As the task employed by Williams et al. ${ }^{3}$ was anticipatory in nature they concluded their findings may be due to the defensive players engaging in anticipatory tasks continuously throughout a match to read opponents intentions and put themselves in the best position for a successful challenge or interception. However, they reasoned this may be less important for offensive players whose goal is typically trying to read the play to set up the best attacking options for the team. All players in netball play a role in attacking and defending, however the priorities change according to position or region of the court. As a result, experienced defenders, even though they may have fewer attacking opportunities than the centre court players and goalers, they may have been exposed to enough opportunities to engage in relevant decision making activities and thus develop appropriate cognitive strategies, even though their main focus is defensive in nature. Interestingly, although not significant, the skilled defenders performed more poorly than the skilled goalers and centre court players. The task in the current study (offensive decision making) may have provided the goalers and centre court players an advantage over defenders as they engage in more offensive decision making during matches. A key responsibility of a defender is to intercept opposition passes which requires a sound reading of the play as it unfolds toward the defender. However, the current task, while presenting the defenders with scenarios from their end of the court, 
were attacking pass options rather than off-the-ball interception tasks and so inadvertently may have reduced the expert advantage for the defensive group.

The encoding specificity hypothesis states that "what is stored is determined by what is perceived and how it is encoded, and what is stored determines what retrieval cues are effective in providing access to what is stored" ${ }^{\prime 12}$ (p.353). Catteeuw et al. ${ }^{7}$ demonstrated that soccer officials were most accurate on the tasks they were most familiar with (referees on the foul detection task and assistant referees on the offside decision making task) indicating a difference in the encoding and retrieval of information between the officials for the different tasks. The current study employed a decision making task, something which all netballers experience each match and most training sessions, albeit some players to a greater extent than others. Our results indicated that skilled players were able to transfer the knowledge they have gained from within their highly specialised playing position to a less familiar positional court area due to similar encoding and retrieval processes, although in some cases they were not able perform at quite the same level of performance as the players who were most familiar with that playing area. The encoding processes between positions may be similar as indicated by the experienced player's ability, particularly the centre court players, who were able to transfer their decision making skills across all three positional scenarios (see Figure 2). The influence of previous exposure to other positions cannot be discounted. As reported, each positional group had some playing experience in other positions. In fact if position specificity was to emerge it would have been expected that the defending players would have been the positional group most likely to display the effect as they had spent less time playing in other positions relative to centre-court and attacking players. Further exploration of this concept with more tightly controlled sampling of the positional experiences of the participants is considered a logical direction for future research.

\section{Conclusion}

The current study has shown limited support to the theory that decision making skill may be domain specific to a playing position. Skilled goalers and centre court players were more accurate on the scenarios for which they were most familiar compared to the other playing positions (albeit not 
1 significantly better), but this trend was unable to be extended to the skilled defensive players. Further

2 research into the effect of playing position on decision making accuracy should examine other

3 component processes such as gaze behaviour and attempt to shift this work from the laboratory to the

4 field.

5

6

\section{Practical Implications}

7

8

9 South Australia and Netball Australia. positional area to another.

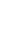

- Decision making is a key discriminator between skilled and lesser skilled performers and consequently should be a key element in skill training programs.

- Coaches can expect a player's perceptual-cognitive skills to be transferable if moved from one

- Position specificity in netball may be more likely to emerge in perceptual-motor skills such as pass interception skill than perceptual-cognitive skills such as offensive decision making.

Acknowledgements

Support for this project was provided through the Australian Institute of Sport, University of 7 
3 1. Hodges NJ, Starkes J, L, MacMahon C. Expert performance in sport: A cognitive 4 perspective. In: Ericsson KA, Charness N, Feltovich PJ, Hoffman RR, editors. The cambridge

5 handbook of expertise and expert performance. New York: Cambridge University Press; 6 2006. p. $471-488$.

7 2. Pyne DB, Gardner AS, Sheehan K, et al. Positional differences in fitness and

8 anthropometric characteristics in Australian football. J Sci Med Sport 2006;9(1-2):143-150.

9 3. Williams AM, Ward P, Ward JD, et al. Domain specificity, task specificity, and expert 10 performance. Res $Q$ Exerc Sport 2008;79(3):428-433.

11 4. Catteeuw P, Helsen W, Gilis B, et al. Decision-making skills, role specificity, and 12 deliberate practice in association football refereeing. J Sports Sci 2009;27(11):1125-1136.

13 5. Raab M. Think SMART, not hard - a review of teaching decision making in sport 14 from an ecological rationality perspective. Phys Educ \& Sport Pedagog 2007;12(1):1-22.

15 6. Ericsson KA, Krampe R, Tesch-Römer C. The role of deliberate practice in the 16 acquisition of expert performance. Psychol Rev 1993;100(3):363-406.

17 7. Ericsson KA, Kintsch W. Long-term working memory. Psychol Rev 1995;102(2):21118245.

19 8. Johnson JG, Raab M. Take the first: Option-generation and resulting choices. Organ 20 Behav Hum Decis Process 2003;91(2):215-229.

219 Griffin L, Mitchell S, Oslin J. Teaching sport concepts and skills: A tactical games 22 approach. Champaign, IL: Human Kinetics; 1997.

23 10. Jones C, Farrow D. The transfer of strategic knowledge: a test of the games 24 classification curriculum model. Bull Phys Educ 1999;35(2):103-123. 
1 11. Mitchell SA, Oslin JL. An investigation of tactical transfer in net games. Eur J Phys

2 Educ 1999;4(2):162-172.

3 12. Abernethy B, Baker J, Côté J. Transfer of pattern recall skills may contribute to the

4 development of sport expertise. Appl Cogn Psychol 2005;19:705-718.

5 13. Smeeton NJ, Ward P, Williams AM. Do pattern recognition skills transfer across

$6 \quad$ sports? A preliminary analysis. J Sports Sci 2004;22:205-213.

7 14. Helsen WF, Pauwels JM. The relationship between expertise and visual information

8 processing in sport. In: Starkes J, Allard F, editors. Cognitive issues in motor expertise.

9 Amsterdam: Elsevier Science Publishers B. V.; 1993. p. 109-134.

10 15. Pallant J. SPSS Survival Manual. 3rd ed. Crows Nest: Allen \& Unwin; 2007.

11 16. Starkes JL. Skill in field hockey: The nature of cognitive advantage. J Sport Psychol $12 \quad 1987 ; 9: 146-160$.

13 17. Tenenbaum G, Yuval R, Elbaz G, et al. The relationship between cognitive 14 characteristics and decision making. Can J App Phys 1993;18(1):48-62. 
2

3 Figure 1. Field set-up of scenario filming

4 Figure 2. Effect of group on response accuracy for scenario type.

5 Figure 3. Effect of group on response accuracy.

6 


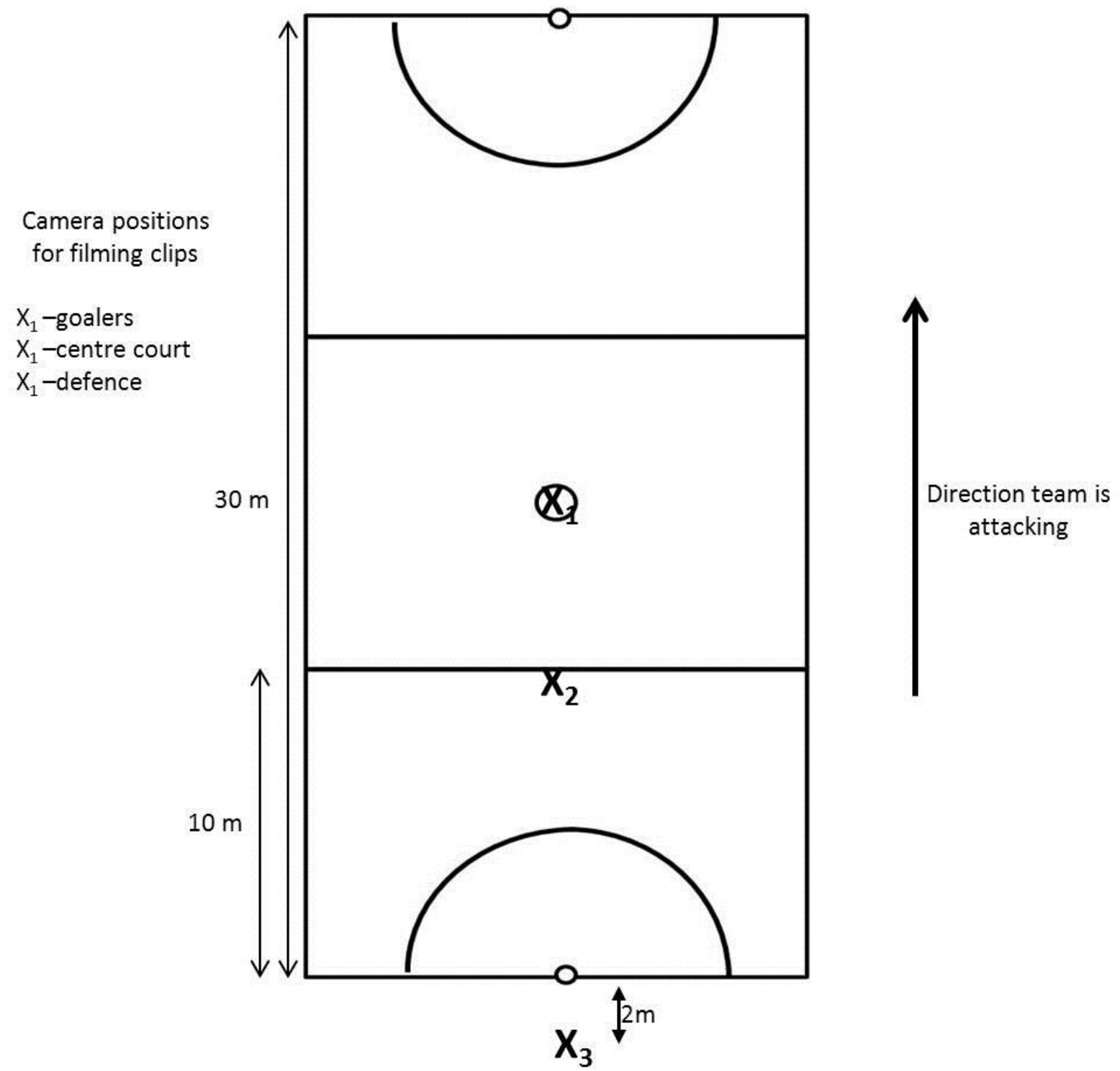

1

2 


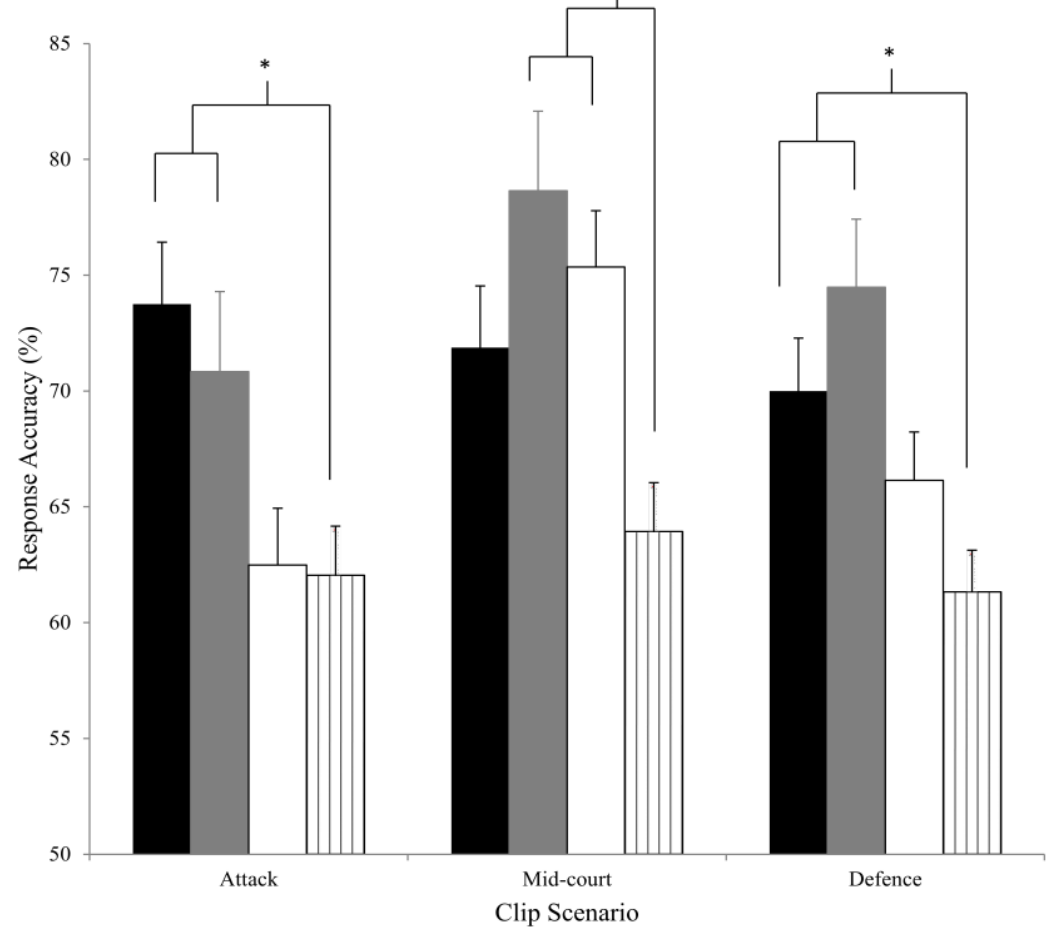

- Skilled Goalers - Skilled Centre Court $\square$ Skilled Defenders $\square$ Novices

$2 * p<.05$. Error bars are representative of the standard error.

3 


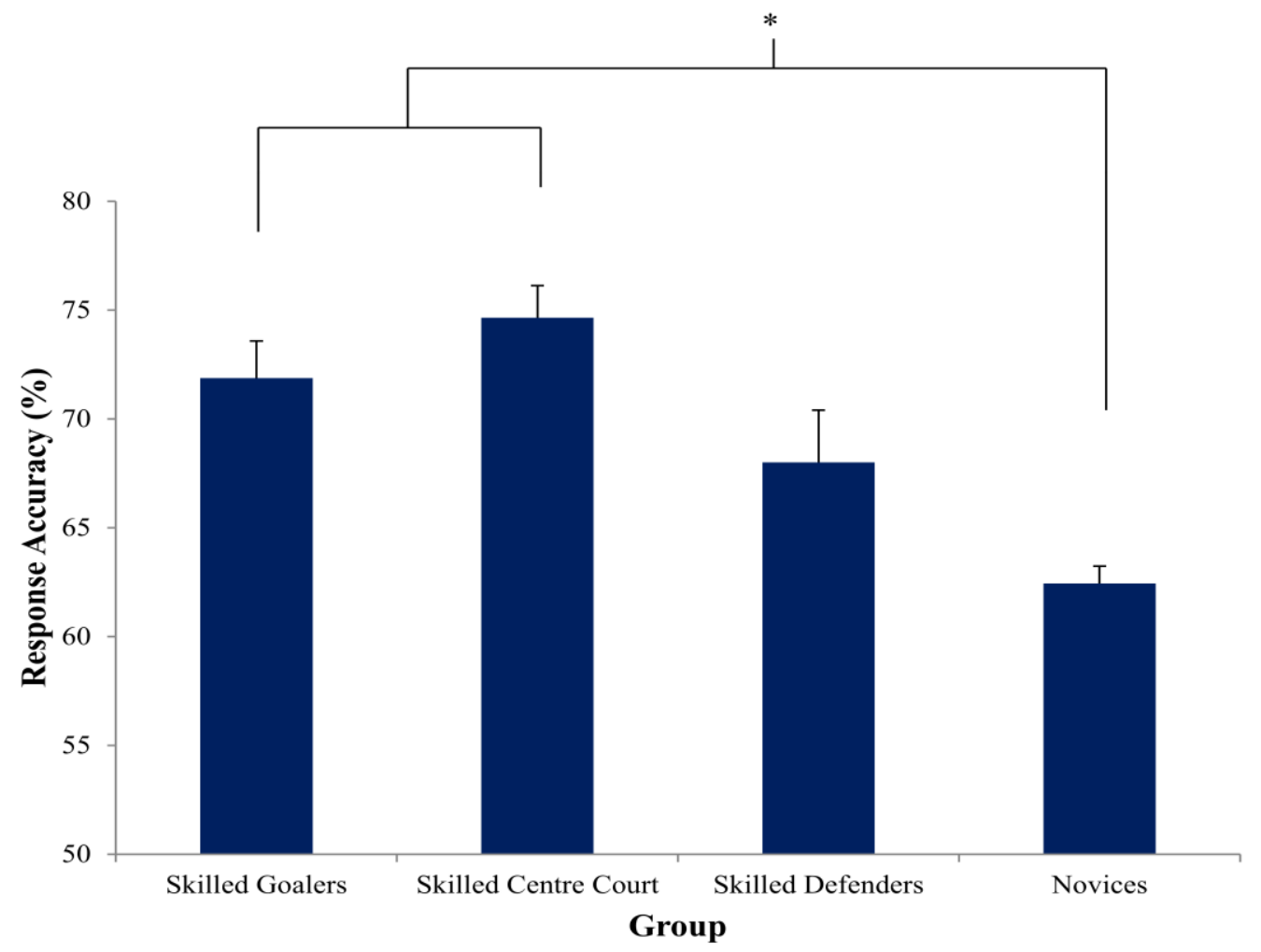

1

$2 * p<.01$. Error bars are representative of the standard error. 